\title{
A Nomogram for Predicting Hospital Mortality in Intensive Care Unit Patients with Acute Myocardial Infarction
}

\author{
Liao $\operatorname{Tan}(1)^{1,2}$ \\ Qian $X u^{3}$ \\ Ruizheng Shi ${ }^{2}$ \\ 'Department of Cardiology, The Third \\ Xiangya Hospital, Central South \\ University Changsha, Hunan, People's \\ Republic of China; ${ }^{2}$ Department of \\ Cardiovascular Medicine, Xiangya \\ Hospital, Central South University, \\ Changsha, Hunan, People's Republic of \\ China; ${ }^{3}$ Department of Cardiovascular \\ Surgery, Xiangya Hospital, Central South \\ University, Changsha, Hunan, People's \\ Republic of China
}

Background: This study aims to construct and validate an early-stage nomogram for predicting hospital mortality of ICU patients with acute myocardial infarction (AMI), to help clinicians determine the appropriate intervention.

Methods: The primary cohort of 2704 patients diagnosed with acute myocardial infarction in admission records from eICU-Collaborative Research Database (eICU-CRD) v2.0. Univariate logistic regression analysis and multivariate logistic regression analysis were enrolled for the construction of the predictive nomogram. Demographic factors, history of clinical cardiovascular disease, vital signs, the use of vasopressors, urine output, and serum variables in the first 24 hours were included in this analysis. The nomogram was evaluated by performance traits including Harrell's concordance index (C-index) and area under the receiver operating characteristic (AUC) analysis, calibration curve, and decision curve analysis (DCA). The nomogram was validated in a different cohort containing 1026 subjects collected from MIMIC-III Database v1.4. Finally, in order to compare the performance with other classic prediction models, AUC analysis, calibration curve, DCA and accuracy analysis (net reclassification improvement (NRI)) were conducted for three ICU scores in validated cohort.

Results: The nomogram revealed 14 predictors of the first 24 hours derived from univariate and multivariable analyses, including age, history of peripheral vascular disease, atrial fibrillation, cardiogenic shock and cardiac arrest, the use of norepinephrine, urine output, white blood cell (WBC), hemoglobin $(\mathrm{Hb})$, red blood cell $(\mathrm{RBC})$, red cell distribution width (RDW), glucose, bicarbonate and magnesium. The C-index of this nomogram was 0.834 (95\% CI 0.812 to 0.856 ). Then, the result of AUC analysis, the DCA and calibration curve indicated that our nomogram was feasible for clinical prediction. The predictive ability and clinical use of the nomogram were verified in the validated cohort. The AUC analysis of ICU scores showed that the AUC of these score systems was ranged from 0.811 to 0.860 (the AUC of nomogram: 0.885). Moreover, our nomogram also showed a better performance in calibration curve and DCA NRI.

Conclusion: The study presents a prediction nomogram incorporating 14 variables that could help identify AMI patients admitted in ICU who might have a high risk of hospital mortality in the first hospitalized 24 hours. This nomogram showed a better performance than normal ICU score systems.

Keywords: prediction, acute myocardial infarction, ICU, nomogram, hospital mortality

\section{Introduction}

Due to the progress of therapy strategies in acute myocardial infarction (AMI), the hospital mortality rate in AMI decreases by years and ranges from $2.5 \%$ to $8 \%$. $^{1-5}$ However, the huge death tolls caused by the high incidence rate of AMI still bring
Department of Cardiovascular Medicine, Xiangya Hospital, Central South University, 88 Xiangya Road, Changsha, 410013, People's Republic of China Email xyshiruizheng@csu.edu.cn 
heavy medical burden in the world. ${ }^{6,7}$ Moreover, a large retrospective cohort study of AMI elderly patients showed the hospital mortality of the patients admitted to ICU is significantly higher than non-ICU patients $(14.3 \%$ vs $8.3 \%) .{ }^{8}$ And more severe complications and death occurred in the first 24 hours in ICU. However, earlystage risk assessment using simple bedside observations in the ICU is lacking. In this situation, an effective predictive model based on early-stage examinations for monitoring and evaluating hospital mortality of ICU patients needs to be constructed, which could help identify highrisk patients in the early-stage and allow rapid triage for cardiac catheterization and reasonable ICU arrangement.

The main existing predictive score models for mortality risk following AMI are the Thrombolysis in Myocardial Infarction (TIMI) score and the Global Registry of Acute Cardiac Events (GRACE) scores. ${ }^{8}$ These scores allow for risk stratification, high-risk identification, and treatment adjustment. However, drawbacks and flaws of the score systems should not be overlooked. Firstly, the TIMI risk score is mainly applied to ST-elevation myocardial infarction (STEMI) and is proved to have low effectiveness in nonSTEMI (NSTEMI) comparatively. Moreover, intraoperative variables are included in these models, so that they are not suitable for evaluation in an early stage. Finally, the clinical utility of these models in ICU patients remains elusive. In addition, the most available scoring systems for survival after ICU admission, including Simplified Acute Physiology Score II (SAPS II), Oxford Acute Severity of Illness Score (OASIS) and the sepsis-related organ failure assessment score (SOFA), have been proved to have limited efficiency, in particular myocardial infarction admission. Too many items also limit the usage in early stages of admission. Hence, a large-scale multicenter study is required to construct a new early-stage prediction model for hospital mortality of AMI patients in ICU.

\section{Methods}

\section{Database Source}

Data of training cohort were collected from eICUCollaborative Research Database (eICU-CRD) v2.0. ${ }^{9}$ Data of validation cohort were obtained from MIMIC-III Database v1.4. eICU-CRD includes 200,859 ICU admissions of 139,367 patients in 2014 and 2015 from 208 hospitals of the United States. MIMIC III includes 61,532 ICU admissions of 46,476 patients from 2001 to
2012 at the Beth Israel Deaconess Medical Center in Boston, MA, USA.

The construction of these two databases was approved by the ethical standards of the institutional review board of the Massachusetts Institute of Technology. Thus, consent was obtained for the original data collection including data in this study.

All data presented in this article were extracted by author Tan, who finished the training course of the National Institutes of Health and acquired the admission (certification number: 35950815). Data extraction was performed using PostgreSQL tools. This study was conducted following the STrengthening the Reporting of OBservational studies in Epidemiology (STROBE) statement. ${ }^{10}$

\section{Study Population and Data Stratification Method}

We selected the patients diagnosed with AMI in the admission records. The patients with multiple admission records and malignant tumors were excluded. Moreover, the patients with censored data were also excluded.

The following information about first $24 \mathrm{~h}$ was extracted: gender, age, ethnicity, admission cardiovascular complications (congestive heart failure, valvular disease, peripheral vascular disease, hypertension, diabetes, hypercholesterolemia, prior myocardial infarction, atrial fibrillation, cardiogenic shock, cardiac arrest), vital signs (systolic blood pressure (SBP), mean blood pressure (MBP) and diastolic blood pressure (DBP)), use of vasopressors (norepinephrine, dopamine, epinephrine), urine output of first $24 \mathrm{~h}$, serum laboratory variables (TnT, CKMB, and white blood cell (WBC), platelet (PLT), red blood cell (RBC), hemoglobin $(\mathrm{Hb})$, red cell distribution (RDW), blood urea nitrogen (BUN), creatinine, glucose, potassium, sodium, bicarbonate and magnesium), and hospital mortality. The serum laboratory variables measured during the first $24 \mathrm{~h}$ of ICU admission were used in this study. If variables were measured multiple times in the first $24 \mathrm{~h}$, the first level was used in this study. Records with missing variables $>10 \%$ were excluded. As for Records with missing variables $\leq 10 \%$, we used the multiple imputation method to deal with the missing data.

\section{Statistical Analysis}

Statistical analyses were conducted by R software (Version 4.1.0; https://www.R-project.org) and Empowerstats 2.0. A 2-sided probability value of $\mathrm{P} \leq 0.05$ was considered 
statistically significant. Continuous variables conforming to a normal distribution were expressed by mean $\pm \mathrm{SD}$ values, and other continuous variables were expressed as median (25th-75th percentile) values. Numbers and percentages were used to present categorical variables. Significant variables in the univariate logistic regression analysis were selected to conduct the multivariate regression analysis in which variables with $\mathrm{P}<0.05$ were considered as possible predictors. Moreover, before we constructed the predicted nomogram, multicollinearity check (VIF step screening methods) and LASSO regression were performed based on these possible variables. Meanwhile, smooth curve fitting and threshold effect analysis were conducted on all significant continuous variables. Finally, a predictive nomogram was constructed in the training cohort based on multivariate analysis.

Nomogram is a kind of prediction tool, through constructing a multifactorial regression model (commonly used Cox regression or Logistic regression), assigning scores to each value level of each variable according to the contribution degree (the size of regression coefficient) of each variable in the model to the outcome index, and then summing up all the scores to get the total score. Finally, the predicted value of the individual outcome event was calculated by the function conversion relationship between the total score and the probability of the outcome event.

The area under the ROC curve (AUC) was measured to assess the discriminative performance of the prediction nomogram. In order to evaluate the calibration, a calibration curve was generated. Then, the nomogram constructed in the training cohort was further validated in the validated cohort. The performance of the model in terms of discrimination and calibration were assessed in the validation cohort using the same methods described above. Also, decision curve analysis (DCA) was constructed to evaluate the clinical usefulness of the prediction nomogram by quantifying the net benefits at different threshold probabilities. Net reclassification improvement (NRI) was introduced to quantify the gain in predictive accuracy achieved by adding new variables to a list of predictors.

\section{Results}

\section{Patients' Characteristics}

As Figure 1 shows, 2704 patients with AMI selected from eICU-CRD were set as training cohort and 1026 patients selected from MIMIC were set as a validated cohort. All data of patients including demographic, disease, and serum laboratory variables in the two cohorts are listed in Table 1.

\section{Independent Predictors in the Training Cohort}

Variables in Table 1 entered univariate and multivariate logistic regression analyses. The multivariate analyses revealed the following significant risk factors for hospital mortality of patients with AMI: age $(\mathrm{OR}=1.036,95 \% \mathrm{CI}$ 1.024 to 1.048$)$, peripheral vascular disease $(\mathrm{OR}=1.749$, 95\% CI 1.107 to 2.764$)$, atrial fibrillation $(\mathrm{OR}=1.551,95 \%$ CI 1.140 to 2.109), cardiogenic shock (OR $=1.553,95 \%$ CI 1.022 to 2.361$)$, cardiac arrest (OR=3.869, 95\% CI 2.765 to 5.414), use of norepinephrine ( $\mathrm{OR}=2.013,95 \%$ CI 1.386 to 2.923), urine output $>2500 \mathrm{~mL}$ ( $\mathrm{OR}=0.344,95 \%$ CI 0.229 to 0.516 ; Reference: urine output $<100 \mathrm{~mL}), \mathrm{WBC}(\mathrm{OR}=1.019$, 95\% CI 1.004 to 1.034$), \mathrm{Hb}(\mathrm{OR}=1.086,95 \%$ CI 1.004 to 1.174), RBC (OR $=0.730,95 \%$ CI 0.587 to 0.908$)$, RDW $(\mathrm{OR}=1.172,95 \% \mathrm{CI} 1.101$ to 1.127$)$, glucose $(\mathrm{OR}=1.002$, $95 \%$ CI 1.001 to 1.003$)$, bicarbonate (OR $=0.923,95 \%$ CI 0.894 to 0.953 ), magnesium ( $\mathrm{OR}=2.036,95 \%$ CI 1.530 to 2.711) (Table 2). Additionally, after adjusting to all other variables, $\mathrm{Hb}, \mathrm{RBC}$ and $\mathrm{RDW}$ showed a linear correlation with hospital mortality according to the results of the above significant continuous variables. Bicarbonate and magnesium presented a u-bend curve correlated with hospital mortality. All smooth curve fitting results and cut-off values are presented in Supplemental Figure 1.

\section{Prediction Nomogram for Hospital Mortality}

Based on the significant independent predictors that were selected variables with ORs, we constructed a nomogram for predicting the hospital mortality of AMI patients from ICU in the training cohort. All variables were identified to be suitable for model construction based on the results of LASSO regression (Supplemental Figure 2). Each variable was given a score on a scale. These scores were then added to obtain the total score, and a vertical line was dropped down from the total points row to estimate the hospital mortality (Figure 2).

\section{Assessment and Validation of the Prediction Nomogram}

The C-index for the prediction nomogram was 0.834 (95\% CI 0.812 to 0.856 ) for the training cohort and was confirmed to be 0.906 (95\% CI 0.884 to 0.928 ) through 
A

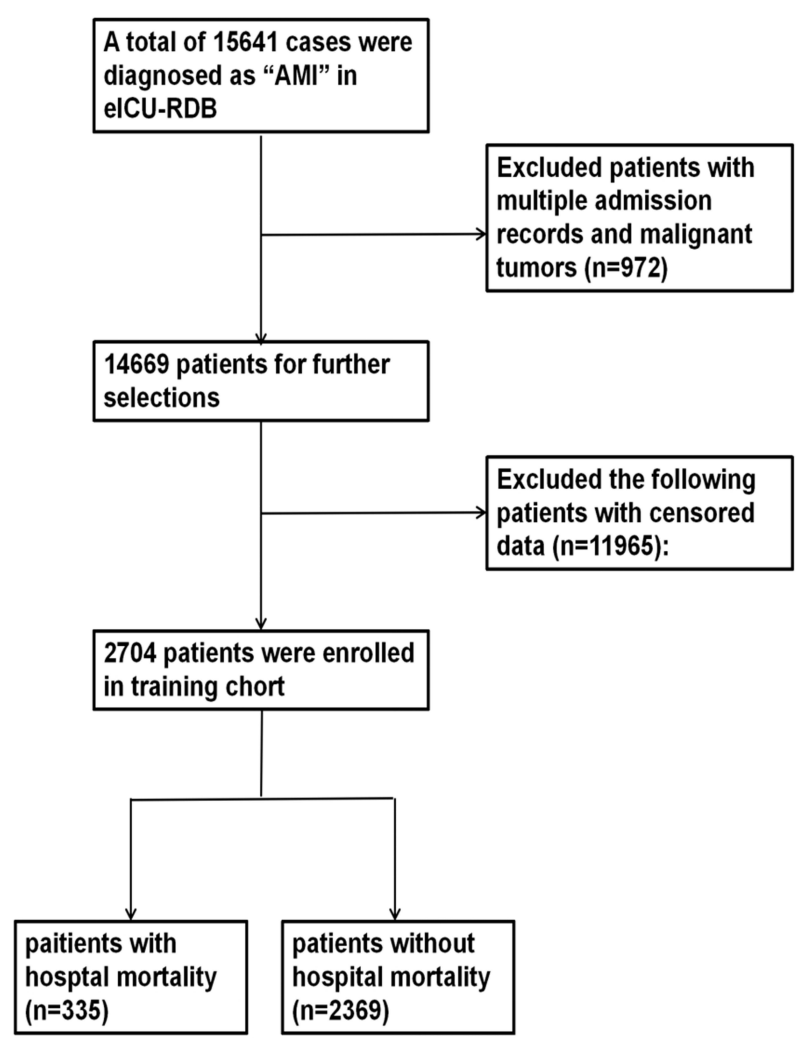

B

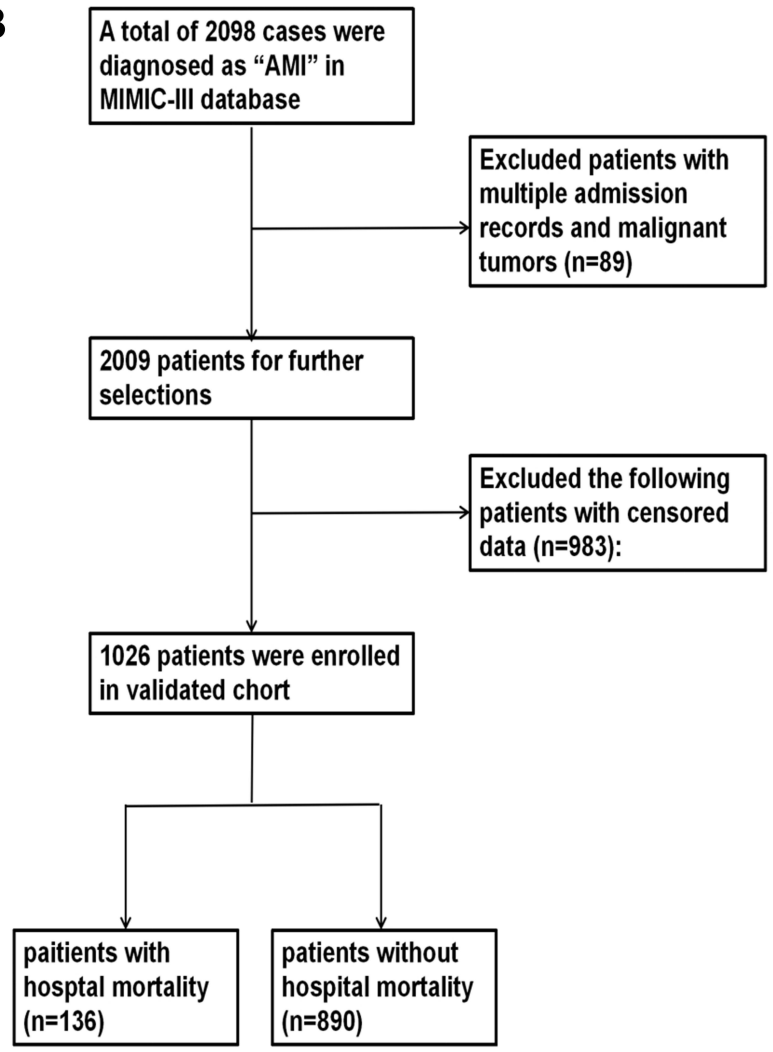

Figure I Flow chart outlining patient selection and grouping process. (A) training cohort selection; (B) validated cohort selection. Abbreviation: AMI, Acute myocardial infarction.

external validation, which indicated the model's good discrimination.

In training and validated cohort, the ROC showed that the resulting model had quite good discrimination (Figure 3), with an AUC of 0.810 and 0.885 , respectively.

The calibration curve of the nomogram for the prediction of hospital mortality risk in ICU patients with AMI demonstrated good agreement in this cohort (Figure 4).

DCA was performed to show the clinical usability and benefits of the nomogram. As shown in Figure 5, DCA curves of the training cohort and validated cohort showed large net benefits across a range of death risks in the validation cohort.

Analysis of accuracy showed that the NRI for the hospital mortality were 0.151 (compared with SAPS II); 0.226 (compared with OASIS); (0.414 (compared with SOFA) in the validation cohort.

Finally, in order to compare the performance with other score systems, ROC, calibration curve and DCA were also conducted on the SAPS II, OASIS and SOFA for the validated cohort. The AUC of these score systems ranged from 0.811 to 0.860 (the AUC of nomogram: 0.885).
Moreover, our nomogram also showed a better performance in calibration curve and DCA analysis (-figure8).

\section{Discussion}

In our study, data were obtained from eICU-CRD and MIMIC-III and followed the same selection criteria. Some characteristic differences existed in these two databases, such as a lower percentage of congestive heart failure, prior myocardial infarction and a higher percentage of cardiogenic shock in MIMIC database, which may result from different patient resources and different admission years. As we mentioned before, eICU-CRD included patients between 2014 and 2015 from 208 hospitals, and patients cohort from eICU-CRD were considered to be wider and more steady and set as training cohort. Meanwhile, MIMIC III included patients from 2001 to 2012 from another single center. Hence, we considered that this cohort was collected from a longer time span and is more suitable to be set as a validated cohort. This kind of setting could guarantee the current usefulness. Then, the univariate and multivariate logistic regression revealed that age, history of peripheral vascular disease, 
Table I Baseline Demographic and Laboratory Characteristics of Patients

\begin{tabular}{|c|c|c|}
\hline Demographic or Characteristics & Training Cohort $(N=2704)$ & Validated Cohort $(\mathrm{N}=1026)$ \\
\hline Age & $65.44 \pm|3.4|$ & $66.91 \pm 13.66$ \\
\hline \multicolumn{3}{|l|}{ Gender } \\
\hline Male & $1715(63.42 \%)$ & 701 (68.32\%) \\
\hline Female & 989 (36.58\%) & 325 (31.68\%) \\
\hline \multicolumn{3}{|l|}{ Ethnicity } \\
\hline Black & $231(8.54 \%)$ & $60(5.85 \%)$ \\
\hline Hispanic & $|4|(5.2 \mid \%)$ & $23(2.24 \%)$ \\
\hline White & 2105 (77.85\%) & 691 (67.35\%) \\
\hline Others & 227 (8.39\%) & $252(24.56 \%)$ \\
\hline \multicolumn{3}{|l|}{ Clinical comorbidities } \\
\hline Congestive heart failure & $670(24.78 \%)$ & $67(6.53 \%)$ \\
\hline Valvular disease & $136(5.03 \%)$ & 18 (I.75\%) \\
\hline Peripheral vascular disease & 164 (6.07\%) & 99 (9.65\%) \\
\hline Hypertension & $1830(67.68 \%)$ & 493 (48.05\%) \\
\hline Diabetes & $649(24.00 \%)$ & $276(26.90 \%)$ \\
\hline Hypercholesterolemia & 181 (6.70\%) & I 42 (I3.84\%) \\
\hline Prior myocardial infraction & $516(19.08 \%)$ & $72(7.02 \%)$ \\
\hline Atrial infibrilation & $442(16.35 \%)$ & 232 (22.6I\%) \\
\hline Cardiogenic_shock & $186(6.88 \%)$ & 184 (17.93\%) \\
\hline Cardiac arrest & $27 \mathrm{I}(10.02 \%)$ & 113 (11.01\%) \\
\hline \multicolumn{3}{|l|}{ Vital signs } \\
\hline SBP & $122.00 \pm 35.75$ & $88.00 \pm 19.25$ \\
\hline MBP & $70.00 \pm 23.00$ & $45.00 \pm 14.00$ \\
\hline DBP & $87.67 \pm 25.00$ & $59.00 \pm 14.67$ \\
\hline \multicolumn{3}{|l|}{ Use of vasoactive agent } \\
\hline Norepinephrine & 231 (8.54\%) & I 83 (I7.84\%) \\
\hline Dopamine & 25 (0.92\%) & 185 (18.03\%) \\
\hline Epinephrine & $36(1.33 \%)$ & 67 (6.53\%) \\
\hline Urine of first $24 \mathrm{~h}$ & $2183.01 \pm 2637.54$ & $2177.02 \pm 1317.82$ \\
\hline \multicolumn{3}{|l|}{ Serum laboratory variables } \\
\hline $\mathrm{TnT}(\mathrm{ng} / \mathrm{mL})$ & $4.87 \pm 5.49$ & $4.76 \pm 5.41$ \\
\hline CKMB (ng/mL) & $|4| .33 \pm|4| .68$ & $154.85 \pm 191.49$ \\
\hline WBC (K/uL) & $14.34 \pm 8.12$ & $14.19 \pm 6.66$ \\
\hline PLT (K/uL) & $237.99 \pm 94.78$ & $259.58 \pm 109.09$ \\
\hline RBC (m/uL) & $3.99 \pm 1.11$ & $3.99 \pm 1.07$ \\
\hline $\mathrm{Hb}(\mathrm{g} / \mathrm{dL})$ & $13.30 \pm 3.20$ & $13.10 \pm 2.80$ \\
\hline RDW (\%) & $14.73 \pm 2.00$ & $14.29 \pm 1.73$ \\
\hline BUN (mg/dL) & $29.52 \pm 21.82$ & $25.79 \pm 18.55$ \\
\hline Creatinine (mg/dL) & $1.79 \pm 1.75$ & $\mid .4 I \pm 1.27$ \\
\hline Glucose(mg/dL) & $198.53 \pm \mid 18.01$ & $198.33 \pm 104.76$ \\
\hline Potassium (mEq/L) & $4.50 \pm 0.75$ & $4.72 \pm 0.86$ \\
\hline Sodium (mEq/L) & $139.57 \pm 4.54$ & $139.67 \pm 3.60$ \\
\hline Bicarbonate (mEq/L) & $25.36 \pm 4.02$ & $24.79 \pm 3.69$ \\
\hline Magnesium(mg/dL) & $2.01 \pm 0.39$ & $2.01 \pm 0.55$ \\
\hline Hospital Mortality & 335 (12.39\%) & 136 (13.26\%) \\
\hline
\end{tabular}




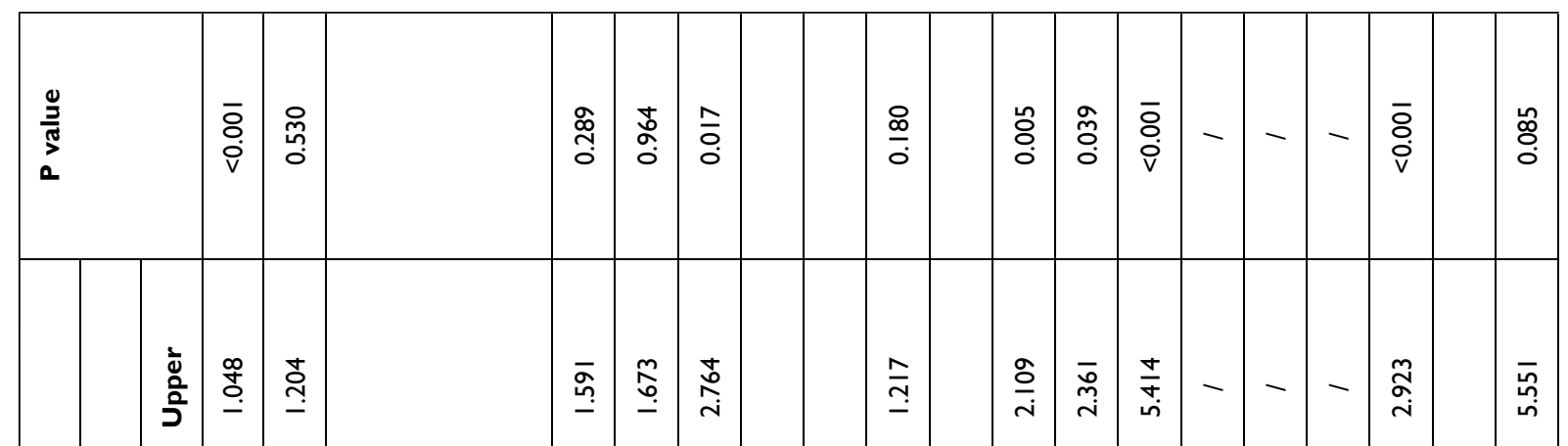

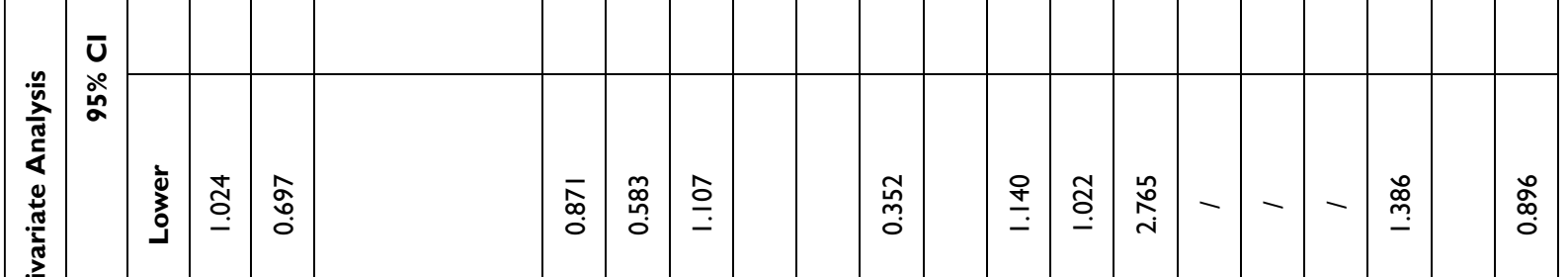

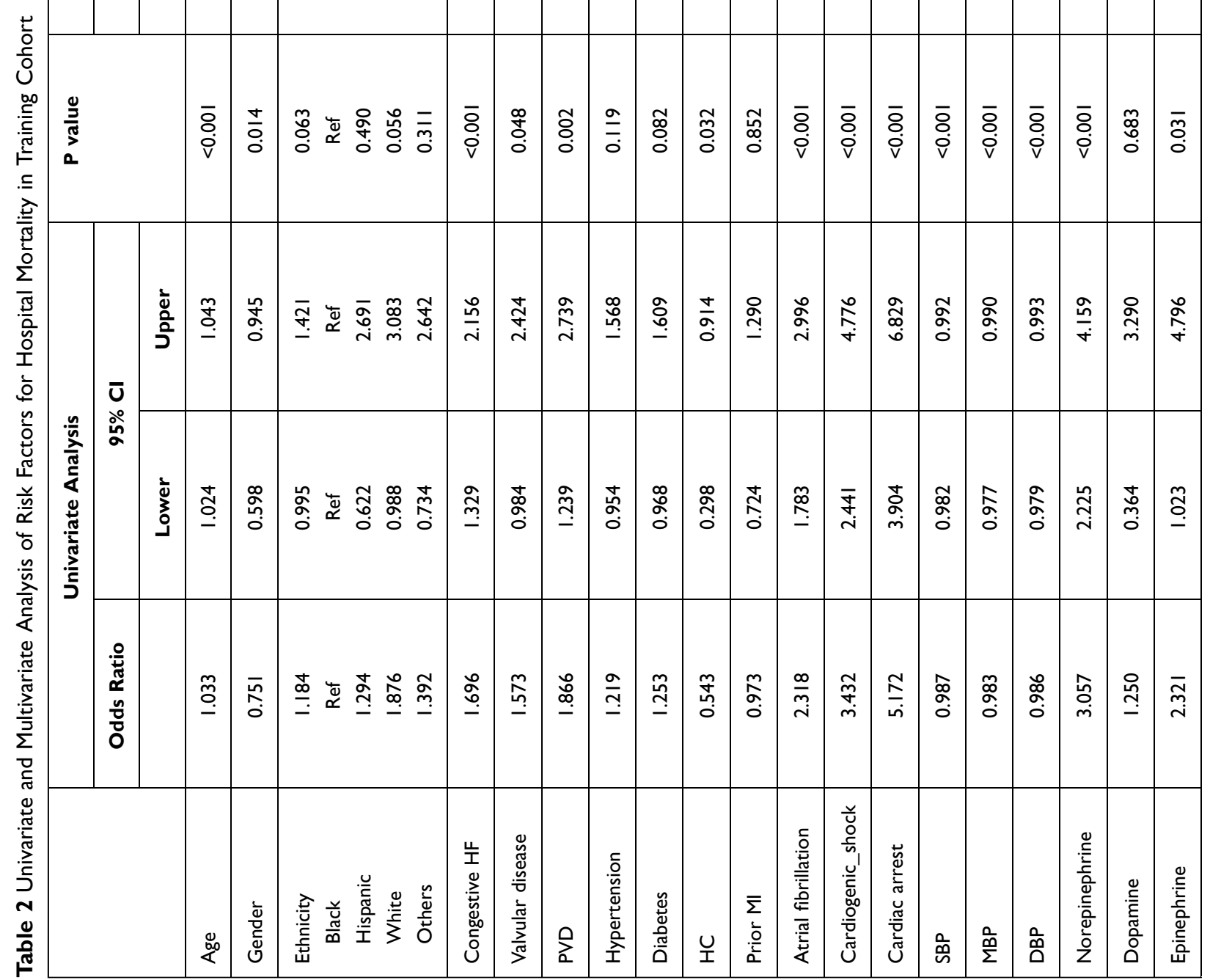




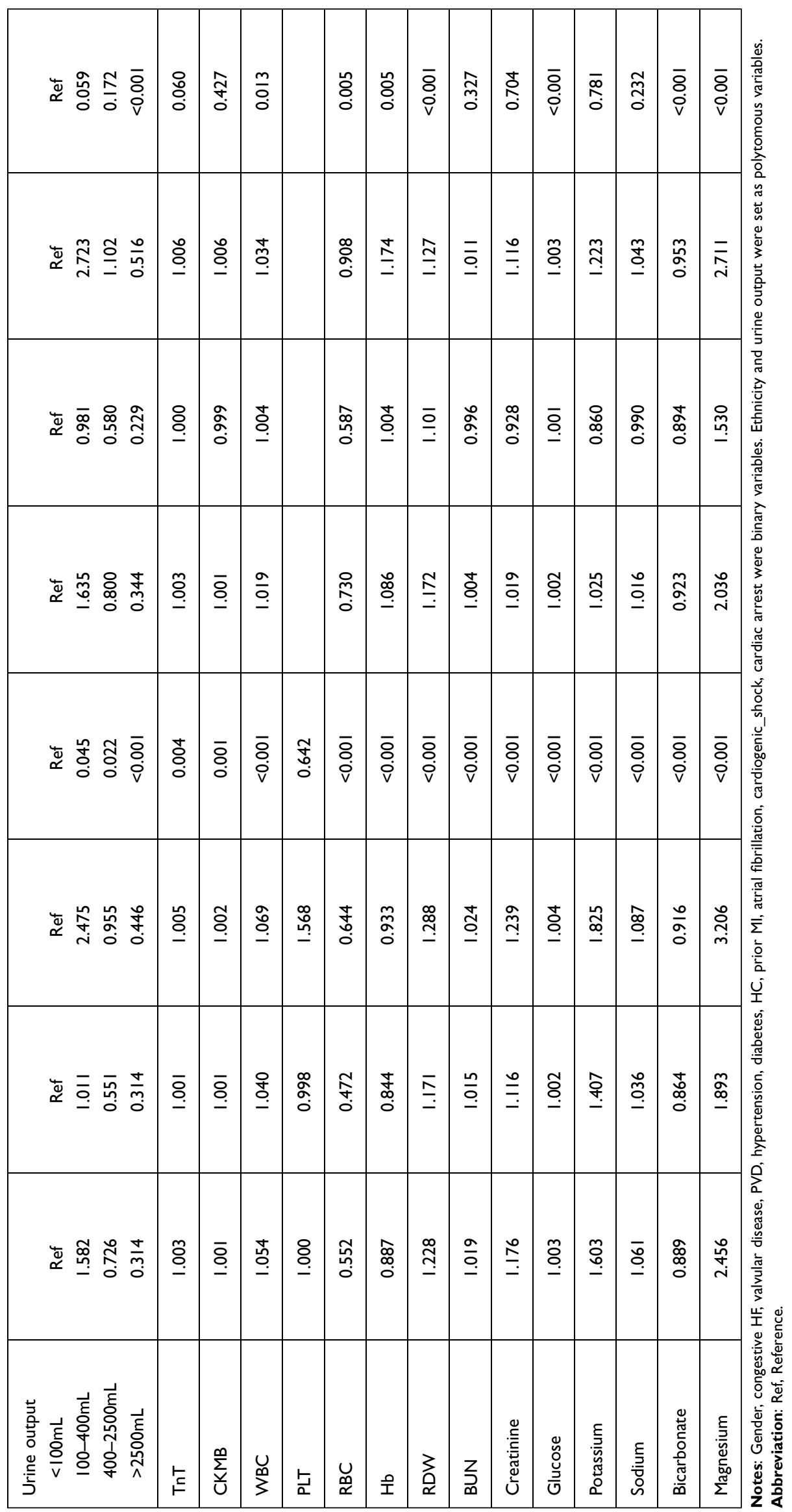


Points

Age(years)

Peripheral vascular disease

Atrial fibrillation

Cardiogenic shock

Cardiac arrest

Norepinephrine

Urine output

WBC(K/uL)

$\mathrm{Hb}(\mathrm{g} / \mathrm{dL})$

$\mathrm{RBC}(\mathrm{m} / \mathrm{uL})$

$\operatorname{RDW}(\%)$

Glucose (mg/dL)

Bicarbonate (mmol/L)

Magnesium (mg/dL)

Total Points

Risk of hospital mortality
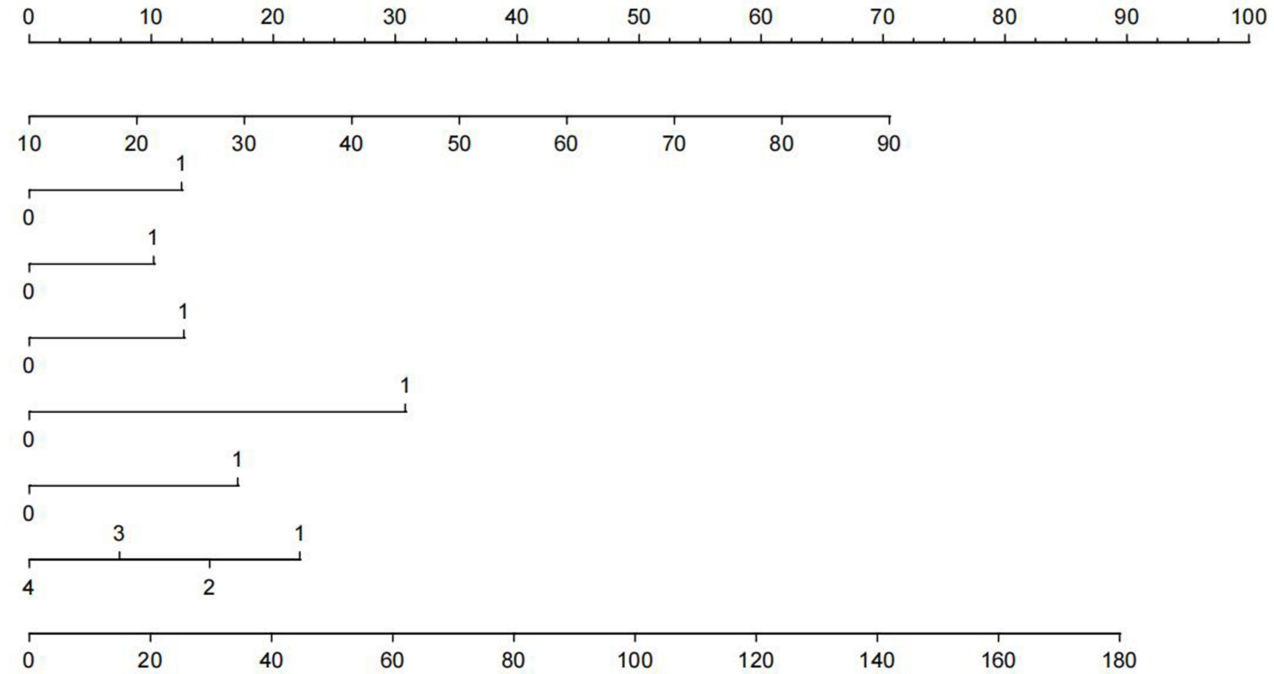

\begin{tabular}{lllllllll}
\hline & 1 & 9 & 11 & 13 & 15 & 17 & 19 & 21
\end{tabular}

$\begin{array}{lllllllllll}6.5 & 6 & 5.5 & 5 & 4.5 & 4 & 3.5 & 3 & 2.5 & 2 & 1.5\end{array}$

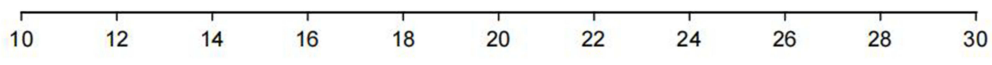

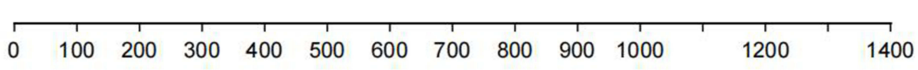

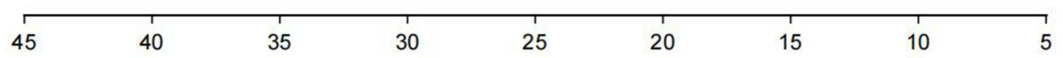

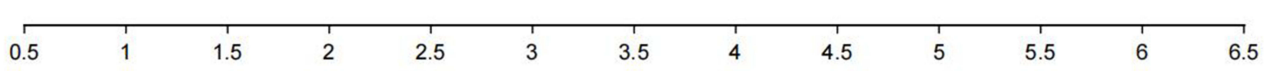

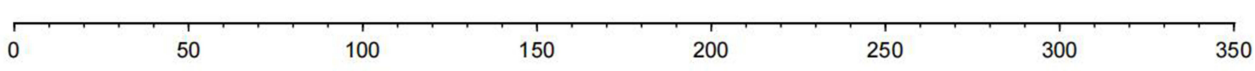

\begin{tabular}{lllll}
\hline 0.1 & 0.3 & 0.5 & 0.7 & 0.9
\end{tabular}

Figure 2 Nomogram for predicting the risk of hospital mortality in AMI patients admitted in ICU. To use this nomogram, first, locate the patient's age, then draw a vertical line straight up to the points axis on the top to get the score associated with age. Repeat the process for the other variables. Add the score of each variable together and locate the total score on the total points axis. Finally, draw a line straight down to the "risk of hospital mortality" axis at the bottom to obtain the probability of hospital mortality. Urine: I: $<100 \mathrm{~mL} ; 2$ : $\geq 100 \mathrm{~mL}$ and $<400 \mathrm{~mL} ; 3: \geq 400 \mathrm{~mL}$ and $<2500 \mathrm{~mL} ; 4:>2500 \mathrm{~mL}$.

atrial fibrillation, cardiogenic shock, cardiac arrest, use of norepinephrine, urine output, WBC, Hb, RBC, RDW, glucose, bicarbonate, and magnesium are independent risk factors for hospital mortality of AMI patients in ICU. Smooth curve fitting and threshold effect analysis were conducted for the above significant continuous variables. According to the identified predictors, we constructed and validated a nomogram for predicting hospital mortality of AMI patients in ICU. In order to identify the performance of the nomogram prediction model, we evaluated it by using several parameters that are commonly assessed in model validations: C-index, ROC curve, calibration curve, DCA and NRI. The results showed great performance in calibration and discrimination both in training and validated cohort. Moreover, the C-index and AUC of the validation cohort were higher than the training cohort, owing to the heterogeneity of the training and validation cohort. Finally, in order to compare the performance with other score systems, ROC, calibration curve and DCA were also conducted on the SAPS II, OASIS and SOFA for the validated cohort.

As for 14 included variables in this nomogram, decreased urine output of first $24 \mathrm{~h}$ and blood electrolyte disturbance (high magnesium and low bicarbonate levels) presented the most critical weights in our nomogram. Firstly, decreased urine output is normally encountered in patients with AMI and associated with increased risk of hospital mortality after AMI. The underlying mechanism could be related to neurohormonal release and activation after AMI, neurohormonal factors, such as renin, ${ }^{11}$ vasopressin, ${ }^{12}$ and endothelin, ${ }^{13}$ and renal endothelial dysfunction, and acceleration of inflammatory cell migration. ${ }^{14}$ Moreover, the underlying mechanism 
A

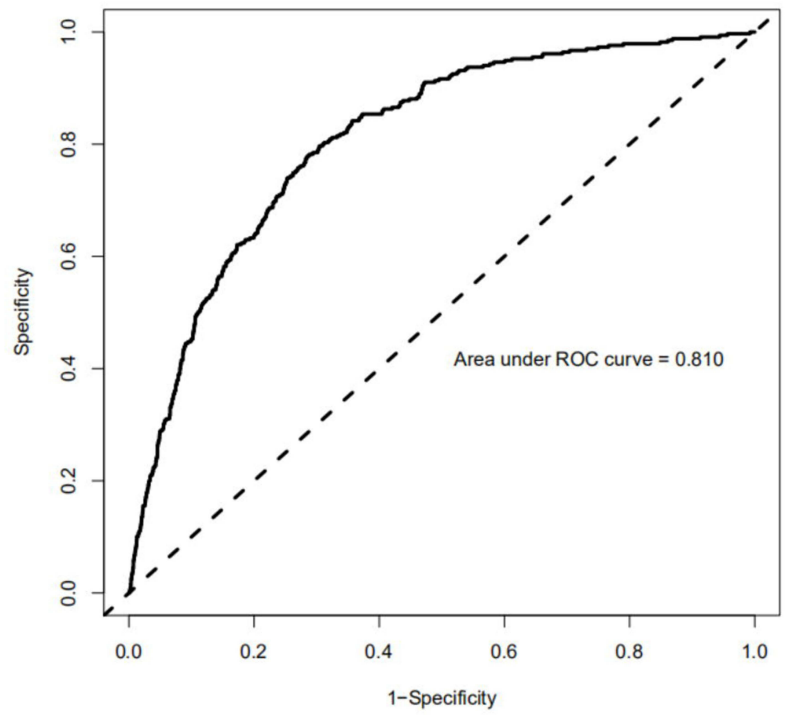

B

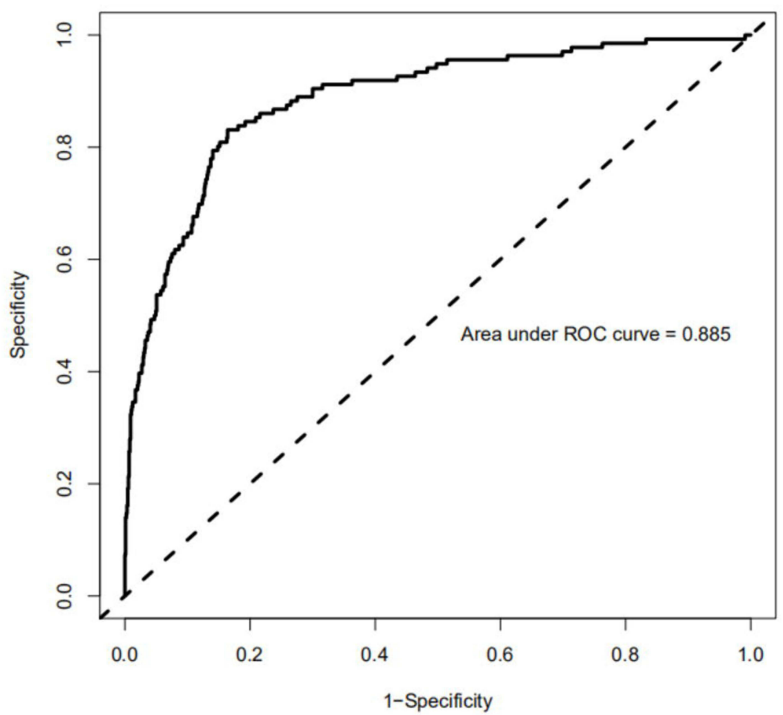

Figure 3 Receiver operating characteristic (ROC) curve of the training and validated cohorts. (A) ROC curve to assess discrimination performance in the training cohort; area under the curve (AUC) was 0.810 . (B) ROC curve for assessing discrimination performance in the validated cohort; AUC was 0.885 .

A

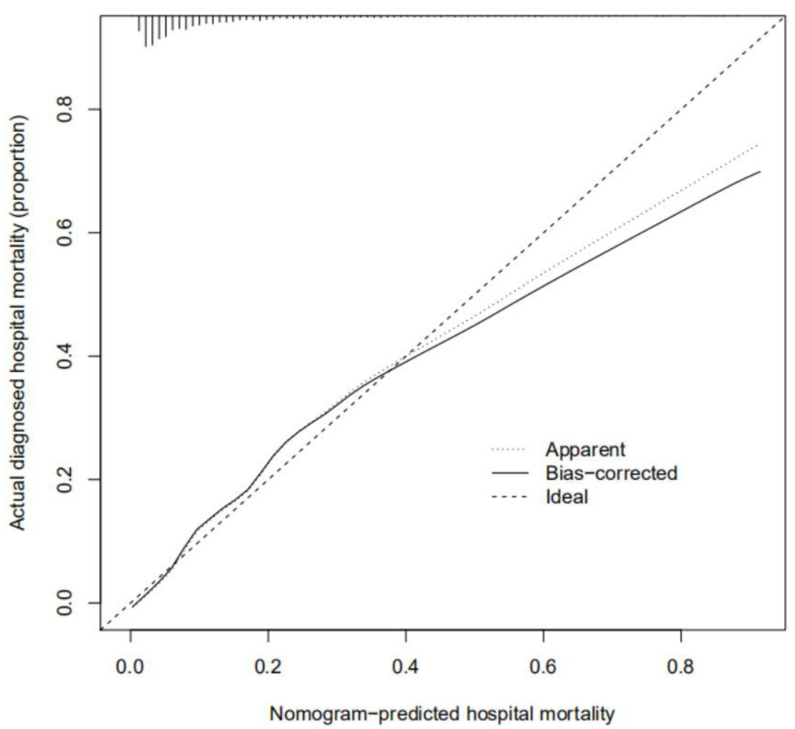

B

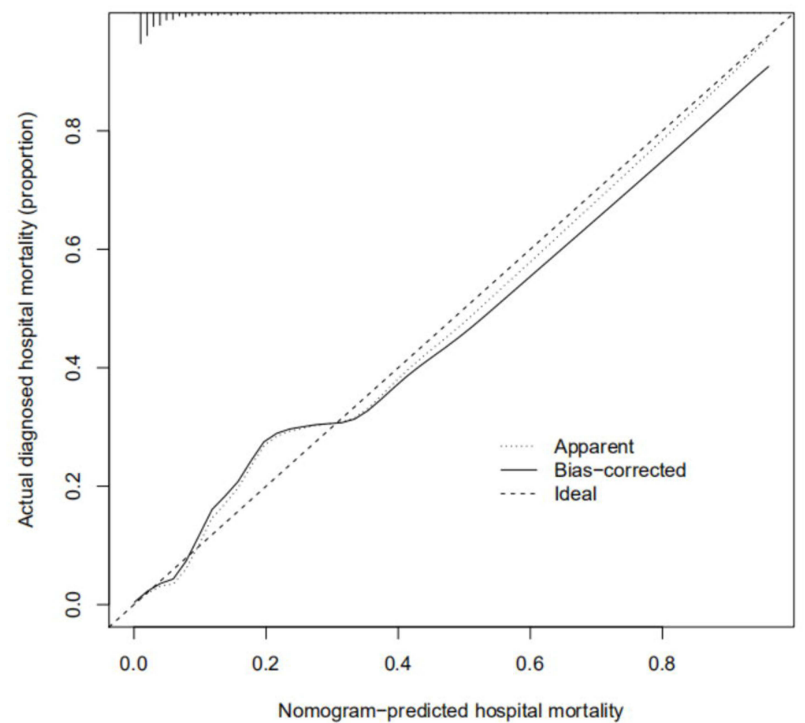

Figure 4 Calibration curves of the training cohort and validated cohort. The $x$-axis represents the nomogram-predicted hospital mortality. The $y$-axis represents the actually diagnosed hospital mortality. The diagonal dotted line represents a perfect prediction by an ideal model. The solid line represents the performance of the nomogram, of which a closer fit to the diagonal dotted line represents a better prediction. (A) calibration curve of training cohort; (B) calibration curve of the validated cohort.

of the association between the hypermagnesemia and high risk of mortality could also be neuromuscular dysfunction, respiratory depression, hypotonia, areflexia, coma and malignant cardiac arrhythmias resulting from hypermagnesemia.${ }^{15,16}$ In addition, low bicarbonate concentration, reflecting underlying metabolic acidosis, has been proven to cause impaired myocardial contractility, and the expression of inflammatory factors in endothelial cells in animal models. ${ }^{17-19}$ These mechanisms can cause poor prognosis in AMI patients but still need clinical validation.

Besides, admission cardiovascular complications were also considered to be strong predictors of mortality (cardiac arrest, cardiogenic shock, atrial fibrillation and peripheral vascular disease). Previous analysis showed that despite a high rate of PCI, patients with cardiac arrest and cardiogenic shock still had an extremely high percentage 
A

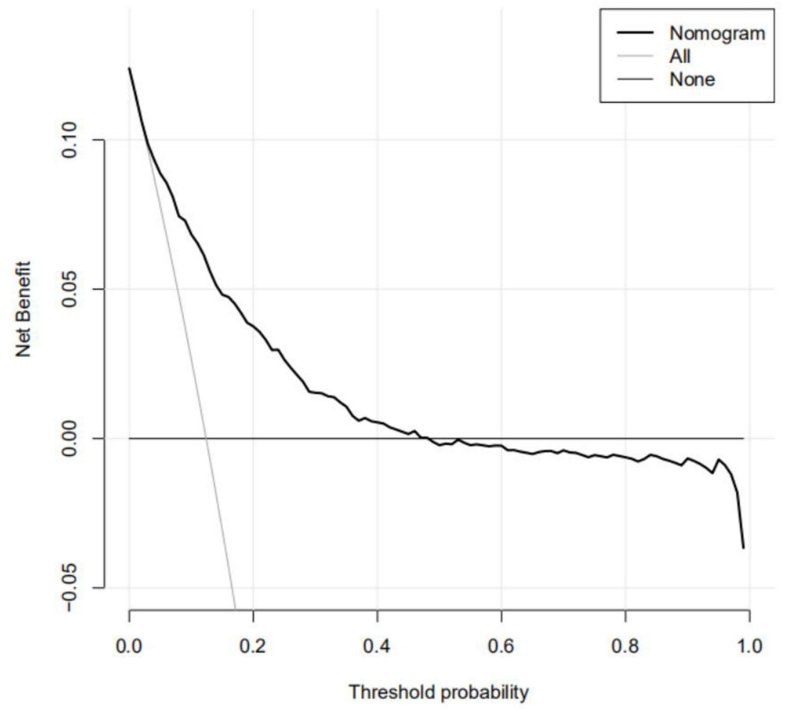

B

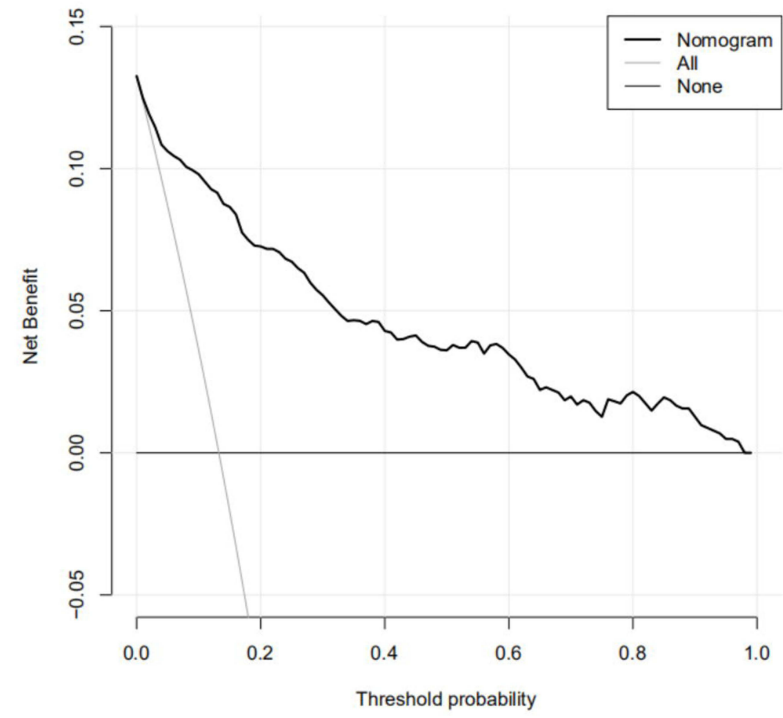

Figure 5 Decision curve analysis (DCA) of the training cohort and validated cohort. The $y$-axis measures the net benefit. The dotted line represents the hospital mortality nomogram. The thin solid line represents the assumption that all patients die in the hospital. The thick solid line represents the assumption that all patients survive in the hospital. (A) DCA of training cohort; (B) DCA of the validated cohort.

of mortality. ${ }^{20}$ This increased mortality is due to higher rates of renal, respiratory failure, stroke and bleeding. Meanwhile, the use of vasoactive agent norepinephrine is closely associated with these kinds of unstable hemodynamics. For atrial fibrillation in the nomogram, the association between the atrial fibrillation and high risk of mortality could mainly be explained by a higher risk of thrombosis and embolism after atrial fibrillation. For the contribution of peripheral vascular disease to poor outcomes in patients with $\mathrm{AMI},{ }^{21-23}$ It is not only attributed to more co-morbidities with peripheral vascular disease but also less aggressive treatment during these patients. ${ }^{22}$

Age and other general blood variables were included and contributed high scores in this nomogram. Age is a wellknown risk factor for mortality of AMI, which may be attributed to more complications and a higher percentage of presenting with atypical symptoms and higher risk of bleeding $^{24}$ that result in poor outcomes. WBC and RDW are identified as normal biomarker of systemic inflammation levels, and are therefore associated with the severity and mortality among patients with AMI. In addition, several possible mechanisms could explain the association between admission of high glucose and AMI mortality. Hyperglycemia could activate platelets and affects fibrinolysis and then lead to thrombogenesis. ${ }^{25,26}$ Moreover, hyperglycemia is associated with the no-reflow phenomenon and endothelium dysfunction. ${ }^{27,28}$ As for the association between
$\mathrm{RBC}$, and mortality, it may be explained that anemia reduces the ability of the heart to increase cardiac output and thereby results in left-ventricle dysfunction. ${ }^{29,30}$ However, the results between the $\mathrm{Hb}$ and mortality showed adverse trends compared with RDW and RBC. In univariate analysis, patients with lower $\mathrm{Hb}$ showed higher mortality risk, but patients with higher $\mathrm{Hb}$ showed higher mortality risk in multivariate analysis. The inclusion of RBC in multivariate analysis counteracts the contribution of anemia and shows the contribution of hyperhemoglobin. Some studies have proved that higher concentrations of $\mathrm{Hb}$ could increase the oxidative insults within the cell and elevate the risk of CVD or mortality. ${ }^{31}$

Due to the lack of TIMI and GRACE scores for AMI in these datasets, the comparison between our nomogram and other cardiovascular score systems was not conducted. However, in a previous study of predicting hospital mortality, the c-index of TIMI score ranged from 0.72 to $0.80(40-42)$. The AUC of TIMI score ranged from 0.73 to $0.83(42,43)$. Similarly, the c-index of GRACE score ranged from to 0.72 and 0.80 and the AUC of GRACE score ranged from 0.71 to $0.82(40,41,44,45)$. In contrast, the C-index is $0.834 / 0.906$ and the AUC is $0.810 / 0.885$ in our nomogram (training cohort/validated cohort). In contrast to previous studies, our prediction nomogram showed better discrimination performance. As an alternative, we compared the performance of our nomogram with regular ICU scores and showed a better performance. 
A

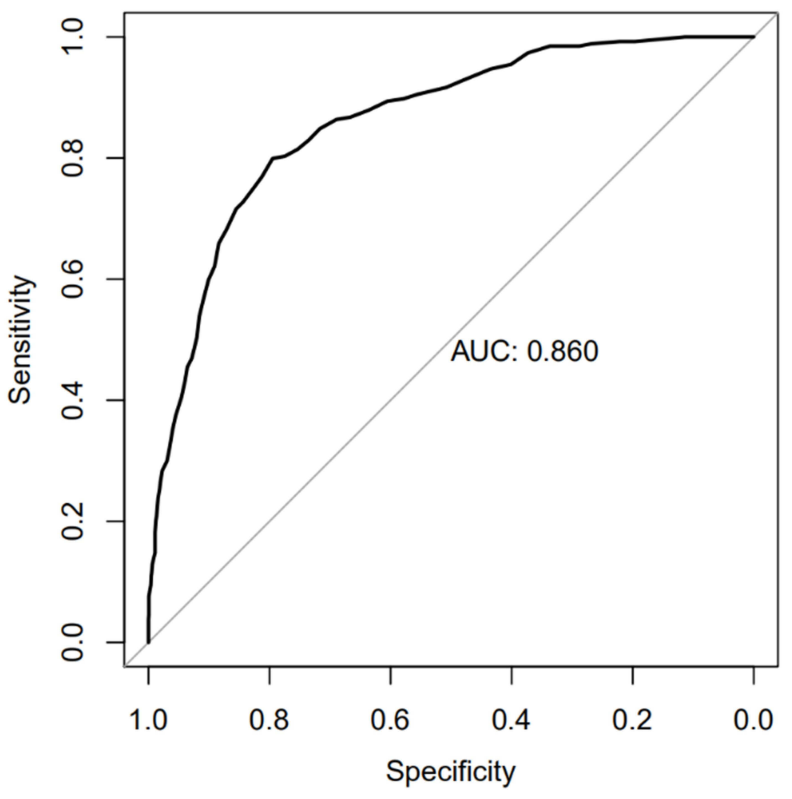

C

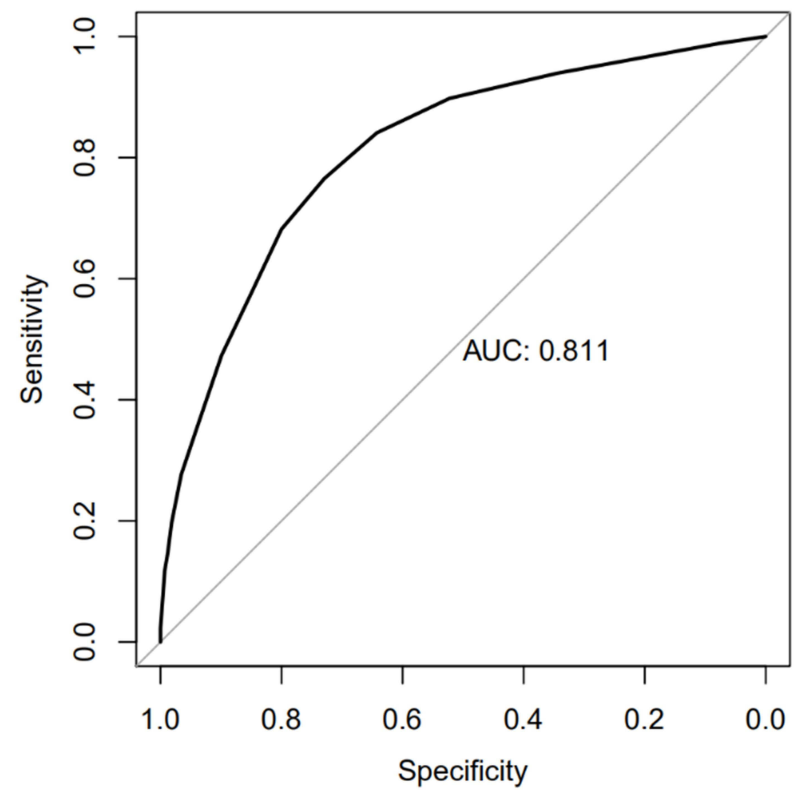

B

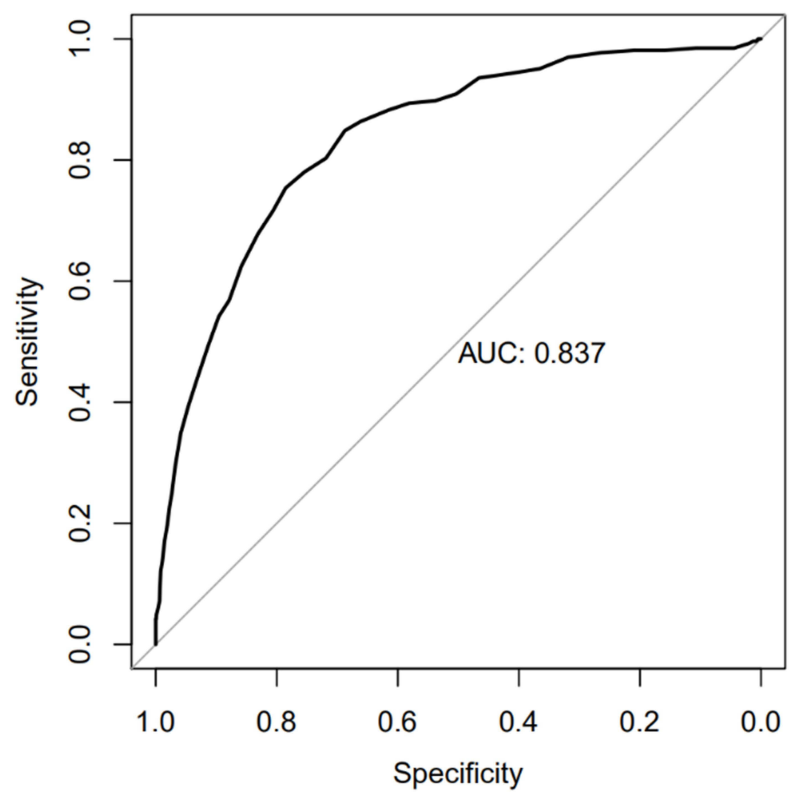

Figure 6 (A) ROC curve of SAPS II to assess discrimination performance in the validated cohort; AUC was 0.860. (B) ROC curve of OASIS to assess discrimination performance in the validated cohort; AUC was 0.837 . (C) ROC curve of SOFA to assess discrimination performance in the validated cohort; AUC was $0.8 \mathrm{II}$.

The strengths of our study lie in its multi-center data collection and a large number of patients included. Our nomogram model contains risk factors collected from medical records within the first 24 hours in the hospital. Most predictors of the model were routinely tested before CABG or PCI, which make it a reality for the physician to calculate the total points and evaluate the risk of hospital mortality within 24 hours after the admission or even before the intervention and to take further preoperative preventive methods to reduce the occurrence of hospital mortality. Additionally, data collected from multi-center and the external validation in our study is crucial to ensure applicability to patients from different hospitals. However, some limits deserve to be mentioned. Firstly, due to the limited resources of data, variables including the time of comorbidity occurrence, revascularization methods and 
A

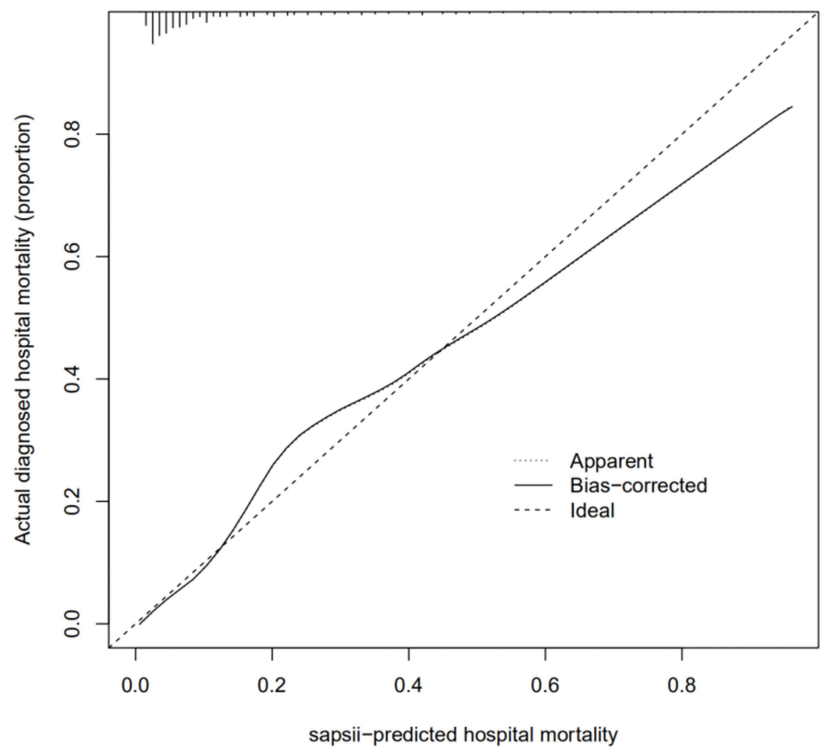

C

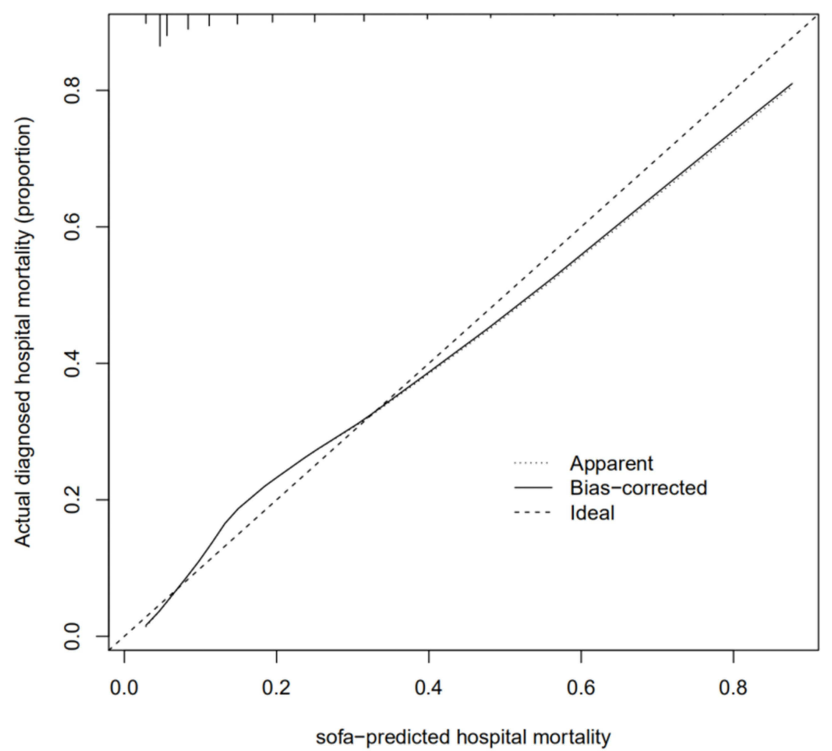

B

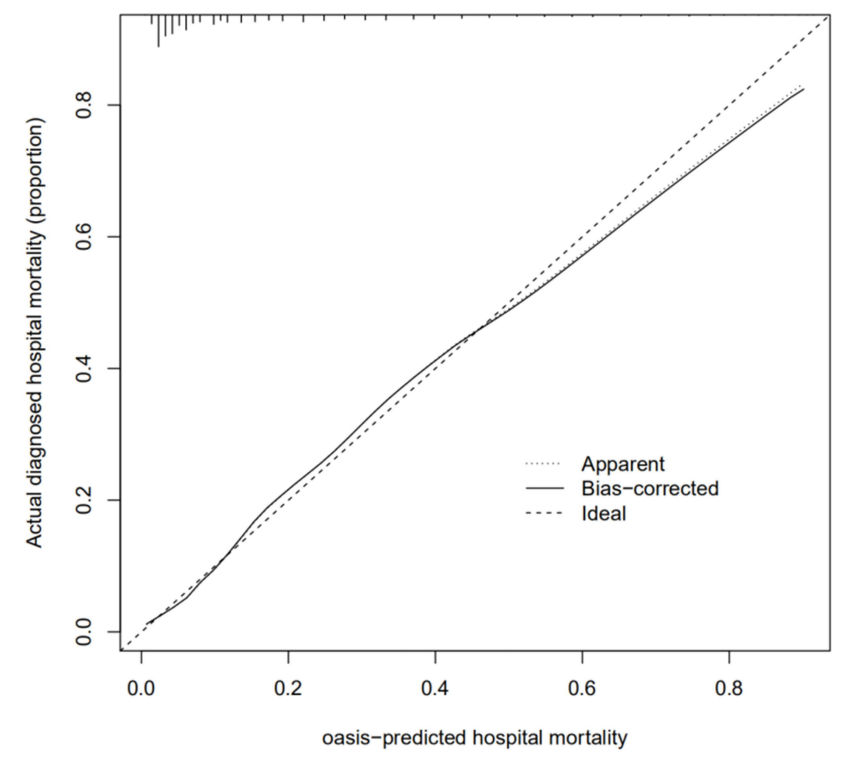

Figure 7 (A) calibration curve of SAPS II of the validated cohort; (B) calibration curve of OASIS of the validated cohort; (C) calibration curve of SOFA of the validated cohort.

novel biomarkers, such as BNP, may lead to potential bias of the results. Moreover, data from eICU-CRD and MIMIC-III were not new enough, and more updated data should be included to validate. Finally, although we constructed a preliminary predicted model and compared our nomogram with the classic score systems, our predicted model still needs further validation and revision according to local condition; further studies addressing the limitations are necessary so that the prediction nomogram could be improved and had a better predicting performance.

\section{Conclusion}

In conclusion, in order to predict the hospital mortality of AMI patients in ICU, we constructed and validated a prediction nomogram based on 2 large, multicenter ICU 
A
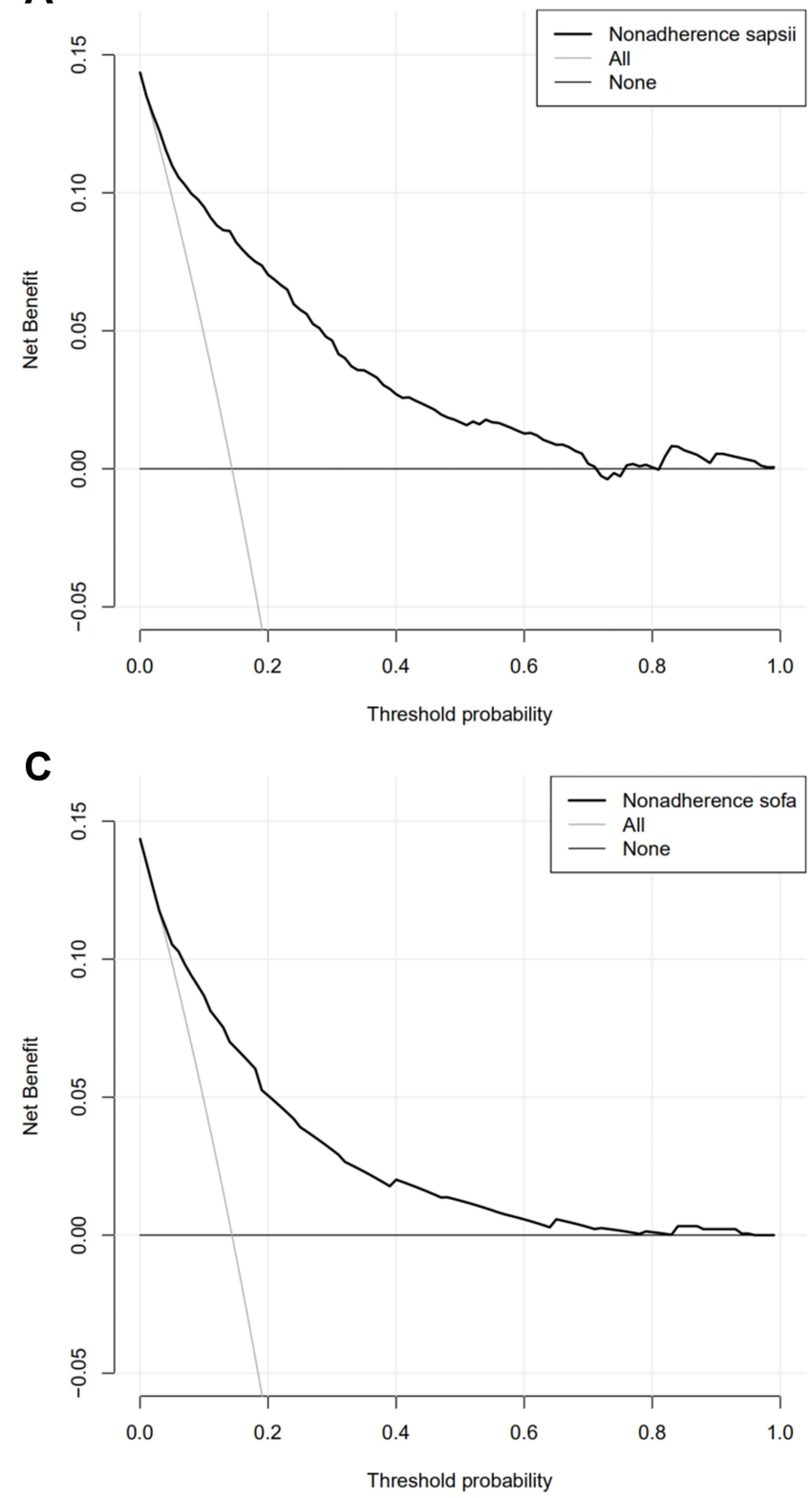

B

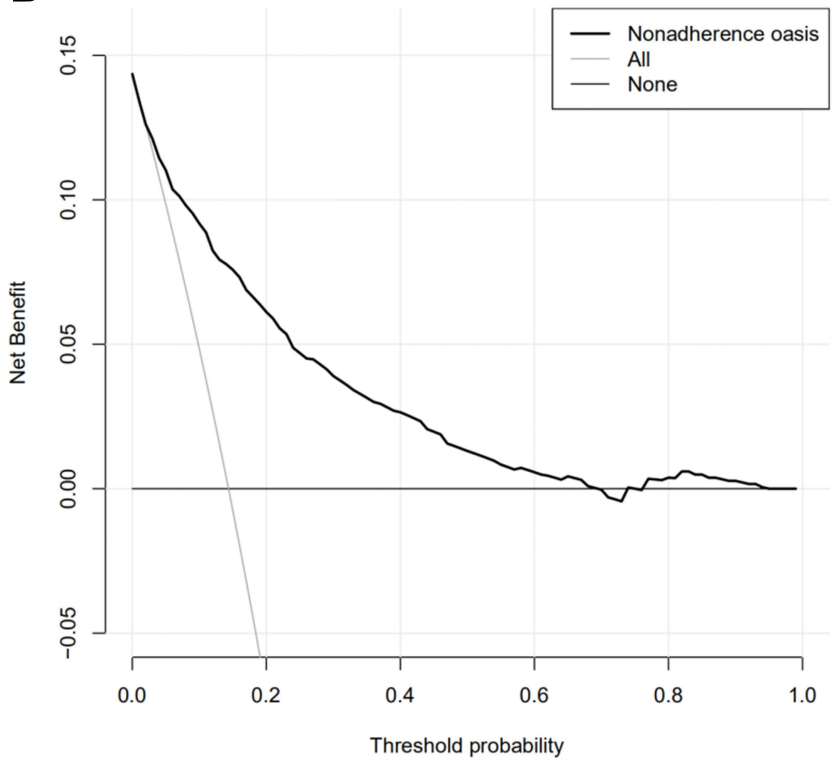

Figure 8 (A) DCA of SAPS II of the validated cohort; (B) DCA of OASIS of the validated cohort; (C) DCA of SOFA of the validated cohort.

databases. The proposed nomogram considered 14 independent risk factors including age, history of peripheral vascular disease, atrial fibrillation, cardiogenic shock and cardiac arrest, use of norepinephrine, urine output, WBC, $\mathrm{Hb}, \mathrm{RBC}, \mathrm{RDW}$, glucose, bicarbonate, magnesium.

\section{Ethical Statement}

The authors are accountable for all aspects of the work, ensuring that questions related to the accuracy or integrity of any part of the work are appropriately investigated and resolved. eICU-CRD and MIMIC III are publicly available databases with anonymized data; ethical review and data sharing statement were approved.

\section{Acknowledgments}

The work was supported by grants from the National Program on Key Basic Research Project of China (Nos. 2019YFF0216304 to S.R.Z.) and the Chinese Cardiovascular Association V.G foundation (Nos.2017CCA-VG-005 to S.R.Z). 


\section{Author Contributions}

All authors contributed to data analysis, drafting or revising the article, gave final approval for the version to be published, agreed to the submitted journal, and agreed to be accountable for all aspects of the work.

\section{Disclosure}

The authors report no conflicts of interest in this work.

\section{References}

1. Rodriguez F, Foody JM, Wang Y, Lopez L. Young Hispanic women experience higher in-hospital mortality following an acute myocardial infarction. J Am Heart Assoc. 2015;4(9):e2089. doi:10.1161/ JAHA.115.002089

2. Harati H, Shamsi A, Firouzkouhi MM, Seyed ZF, Ghazi A. The mortality rate of myocardial infarction patients with and without opium dependent. Int J High Risk Behav Addict. 2015;4(3):e22576. doi:10.5812/ijhrba.22576

3. Rohani A, Akbari V, Moradian K, Malekzade J. Combining white blood cell count and thrombosis for predicting in-hospital outcomes after acute myocardial infraction. J Emerg Trauma Shock. 2011;4 (3):351-354. doi:10.4103/0974-2700.83862

4. Plakht Y, Gilutz H, Shiyovich A. Sodium levels during hospitalization with acute myocardial infarction are markers of in-hospital mortality: Soroka acute myocardial infarction II (SAMI-II) project. Clin Res Cardiol. 2018;107(10):956-964. doi:10.1007/s00392-018-1268-5

5. Dakik HA, Chehab O, Eldirani M, et al. A new index for preoperative cardiovascular evaluation. J Am Coll Cardiol. 2019;73 (24):3067-3078. doi:10.1016/j.jacc.2019.04.023

6. Muller G, Flecher E, Lebreton G, et al. The ENCOURAGE mortality risk score and analysis of long-term outcomes after VA-ECMO for acute myocardial infarction with cardiogenic shock. Intensive Care Med. 2016;42(3):370-378. doi:10.1007/s00134-016-4223-9

7. Valley TS, Sjoding MW, Goldberger ZD, Cooke CR. ICU use and quality of care for patients with myocardial infarction and heart failure. Chest. 2016;150(3):524-532. doi:10.1016/j.chest.2016.05.034

8. Valley TS, Iwashyna TJ, Cooke CR, et al. Intensive care use and mortality among patients with ST elevation myocardial infarction: retrospective cohort study. BMJ. 2019;365:11927. doi:10.1136/bmj.11927

9. Rouleau JL, Packer M, Moye L, et al. Prognostic value of neurohumoral activation in patients with an acute myocardial infarction: effect of captopril. J Am Coll Cardiol. 1994;24(3):583-591. doi:10.1016/0735-1097(94)90001-9

10. Schaller MD, Nussberger J, Feihl F, et al. Clinical and hemodynamic correlates of elevated plasma arginine vasopressin after acute myocardial infarction. Am $J$ Cardiol. 1987;60(14):1178-1180. doi:10.1016/0002-9149(87)90416-4

11. Mitchell JH, Wildenthal K, Johnson RJ. The effects of acid-base disturbances on cardiovascular and pulmonary function. Kidney Int. 1972;1(5):375-389. doi:10.1038/ki.1972.48

12. Brown RJ, Cohen I, Noble D. The interactions of protons, calcium and potassium ions on cardiac Purkinje fibres. $J$ Physiol. 1978;282:345-352. doi:10.1113/jphysiol.1978.sp012467

13. Kontos MC, Scirica BM, Chen AY, et al. Cardiac arrest and clinical characteristics, treatments and outcomes among patients hospitalized with ST-elevation myocardial infarction in contemporary practice: a report from the National Cardiovascular Data Registry. Am Heart J. 2015;169(4):515-522. doi:10.1016/j.ahj.2015.01.010

14. Moscucci M, Kline-Rogers E, Share D, et al. Simple bedside additive tool for prediction of in-hospital mortality after percutaneous coronary interventions. Circulation. 2001;104(3):263-268. doi:10.1161/01. cir.104.3.263
15. Guerrero M, Harjai K, Stone GW, et al. Usefulness of the presence of peripheral vascular disease in predicting mortality in acute myocardial infarction patients treated with primary angioplasty (from the Primary Angioplasty in Myocardial Infarction Database). Am J Cardiol. 2005;96(5):649-654. doi:10.1016/j.amjcard.2005.04.037

16. Pana TA, Wood AD, Mamas MA, et al. Myocardial infarction after acute ischaemic stroke: incidence, mortality and risk factors. Acta Neurol Scand. 2019;140(3):219-228. doi:10.1111/ane.13135

17. Avezum A, Makdisse M, Spencer F, et al. Impact of age on management and outcome of acute coronary syndrome: observations from the Global Registry of Acute Coronary Events (GRACE). Am Heart J. 2005;149(1):67-73. doi:10.1016/j.ahj.2004.06.003

18. Puig DM. Platelet function and hyperglycemia in acute coronary syndrome. Rev Esp Cardiol. 2014;67(1):3-5. doi:10.1016/j.rec.2013.08.011

19. Undas A, Wiek I, Stepien E, Zmudka K, Tracz W. Hyperglycemia is associated with enhanced thrombin formation, platelet activation, and fibrin clot resistance to lysis in patients with acute coronary syndrome. Diabetes Care. 2008;31(8):1590-1595. doi:10.2337/dc08-0282

20. Ege M, Guray U, Guray Y, et al. Relationship between TIMI frame count and admission glucose values in acute ST elevation myocardial infarction patients who underwent successful primary percutaneous intervention. Anadolu Kardiyol Derg. 2011;11(3):213-217. doi:10.5152/akd.2011.055

21. Kanaya AM, Dobrosielski DA, Ganz P, et al. Glycemic associations with endothelial function and biomarkers among 5 ethnic groups: the Multi-Ethnic Study of Atherosclerosis and the Mediators of Atherosclerosis in South Asians Living in America studies. $J \mathrm{Am}$ Heart Assoc. 2013;2(1):e4283. doi:10.1161/JAHA.112.004283

22. Hagl S, Heimisch W, Meisner H, Erben R, Baum M, Mendler N. The effect of hemodilution on regional myocardial function in the presence of coronary stenosis. Basic Res Cardiol. 1977;72(4):344-364. doi:10.1007/BF02023594

23. Geha AS. Coronary and cardiovascular dynamics and oxygen availability during acute normovolemic anemia. Surgery. 1976;80(1):47-53.

24. Stack SW, Berger SA. The effects of high hematocrit on arterial flowA phenomenological study of the health risk implications. Chem Eng Sci. 2009;64(22SI):4701-4706. doi:10.1016/j.ces.2009.07.017

25. Roy A, Sikdar J, Seal P, Haldar R. Cigarette smokers develop structurally modified hemoglobin: a possible way of increasing oxidative stress. Inhal Toxicol. 2015;27(6):300-307. doi:10.3109/ 08958378.2015.1045052

26. Chen YH, Huang SS, Lin SJ. TIMI and GRACE risk scores predict both short-term and long-term outcomes in Chinese patients with acute myocardial infarction. Acta Cardiol Sin. 2018;34(1):4-12. doi:10.6515/ACS.201801_34(1).20170730B

27. Qi Y, Wang W, Zhang K, et al. Development and validation of Women Acute Myocardial Infarction in-Hospital Mortality Score (WAMI Score). Int J Cardiol. 2018;259:31-39. doi:10.1016/j.ijcard.2017.12.010

28. Wu C, Gao XJ, Zhao YY, et al. [Prognostic value of TIMI and GRACE risk scores for in-hospital mortality in Chinese patients with non-ST-segment elevation myocardial infarction]. Zhonghua Xin Xue Guan Bing Za Zhi. 2019;47(4):297-304. [Chinese]. doi:10.3760/cma.j.issn.0253-3758.2019.04.007

29. Wei XB, Liu YH, He PC, et al. Additive prognostic value of left ventricular ejection fraction to the TIMI risk score for in-hospital and long-term mortality in patients with ST segment elevation myocardial infarction. J Thromb Thrombolysis. 2017;43(1):1-6. doi:10.1007/ s11239-016-1407-7

30. De-miguel-balsa E, Latour-Perez J, Baeza-Roman A, Amoros-Verdu C, Fernandez-Lozano JA. GRACE score validation in predicting hospital mortality: analysis of the role of sex. $J$ Womens Health. 2017. doi:10.1089/jwh.2016.5940

31. Yusufali A, Zubaid M, Al-Zakwani I, et al. Validation of the GRACE risk score for hospital mortality in patients with acute coronary syndrome in the Arab Middle East. Angiology. 2011;62(5):390-396. doi: $10.1177 / 0003319710387921$ 


\section{Publish your work in this journal}

The International Journal of General Medicine is an international, peer-reviewed open-access journal that focuses on general and internal medicine, pathogenesis, epidemiology, diagnosis, monitoring and treatment protocols. The journal is characterized by the rapid reporting of reviews, original research and clinical studies across all disease areas. The manuscript management system is completely online and includes a very quick and fair peer-review system, which is all easy to use. Visit http://www.dovepress.com/ testimonials.php to read real quotes from published authors.

Submit your manuscript here: https://www.dovepress.com/international-journal-of-general-medicine-journal 